\title{
RESPUESTA INELÁSTICA DE MARCOS DÚCTILES CON CONTRAVIENTO EXCÉNTRICO
}

\author{
Jesús Salvador García Carrera ${ }^{(1,2)}$, Edgar Tapia Hernández ${ }^{(3)}$
}

\begin{abstract}
RESUMEN
En este artículo se discuten los resultados de análisis inelásticos estáticos y dinámicos de edificios regulares de 6 y 9 pisos estructurados con marcos de acero dúctiles con contraventeo excéntrico. Las estructuras se analizaron mediante modelos detallados en OpenSees. Los resultados señalan que los marcos con contravientos excéntricos de mediana altura diseñados conforme a los principios de capacidad podrían desarrollar un comportamiento indeseable, caracterizados por una concentración de daño. Con el propósito de mejorar el conocimiento adquirido, se evaluaron con detalle los parámetros y capacidades inelásticas del sistema estructural.
\end{abstract}

Palabras clave: Contraviento excéntrico, mecanismo de colapso, sobrerresistencia, ductilidad.

\section{INELASTIC RESPONSE OF DUCTILE ECCENTRICALLY BRACED FRAMES}

\begin{abstract}
In this paper, results of static and dynamic nonlinear analyses of 6- and 9-stories regular buildings structured with eccentrically braced ductile steel frames are discussed. The structures were analyzed through detailed models in OpenSees. The results underlined that mid-rise eccentrically braced structures designed in compliance with capacity design principles can develop undesired behavior, characterized by a damage concentration. In order to improve the knowledge acquired, parameters and inelastic capacities of the structural system were carefully assessed.
\end{abstract}

Keywords: Eccentrically brace, collapse mechanism, overstrength, ductility.

\section{INTRODUCCIÓN}

Los reglamentos vigentes para el diseño sismo-resistente permiten que las estructuras, ante una demanda sísmica intensa, se diseñen para incursionar en el intervalo inelástico. El daño estructural relacionado con esa incursión inelástica permite al sistema disipar energía, que pretende garantizar que la estructura permanezca sin colapso. Las previsiones sobre la disipación se definen desde una etapa de diseño, donde a

Artículo recibido el 27 de febrero de 2017 y aprobado para su publicación el 1 de mayo de 2019. Se aceptarán comentarios y/o discusiones hasta cinco meses después de su publicación.

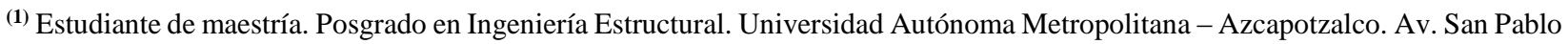
180. Col. Reynosa Tamaulipas, Ciudad de México, C.P. 02200.

(2) Investigador. Instituto Nacional de Electricidad y Energías Limpias, Gerencia de Ingeniería Civil. Reforma 113, Col. Palmira, Cuernavaca, Morelos. C.P. 62490. jsgarcia@iie.org.mx

(3) Profesor - Investigador, Departamento de Materiales, Universidad Autónoma Metropolitana - Azcapotzalco, Av. San Pablo 180. Col. Reynosa Tamaulipas, Ciudad de México, C.P.02200. etapiah@azc.uam.mx 
través de factores y consideraciones se establece y presupone la respuesta inelástica esperada por los sistemas estructurales aplicando los criterios normativos.

Específicamente, en los marcos dúctiles con contraventeo excéntrico, las diagonales generan una excentricidad en las vigas donde se inducen concentraciones de esfuerzos cortantes y momentos flexionantes significativos. Esa zona de la viga, que se denomina eslabón o viga enlace, se diseña para disipar energía mientras que, el resto de los elementos se diseñaran para tener un comportamiento cuasielástico. Así, la premisa fundamental de diseño de un marco contraventeado excéntricamente es que la viga enlace sea un fusible estructural capaz de desarrollar una respuesta uniforme, dúctil y estable. En la figura 1 se muestran los límites normativos (CNBC-05 2005; AISC 341-16 2016; NTC-DCEA-17) que establecen las fronteras para que una viga enlace esté gobernada por los efectos en corte, flexión o efectos combinados.

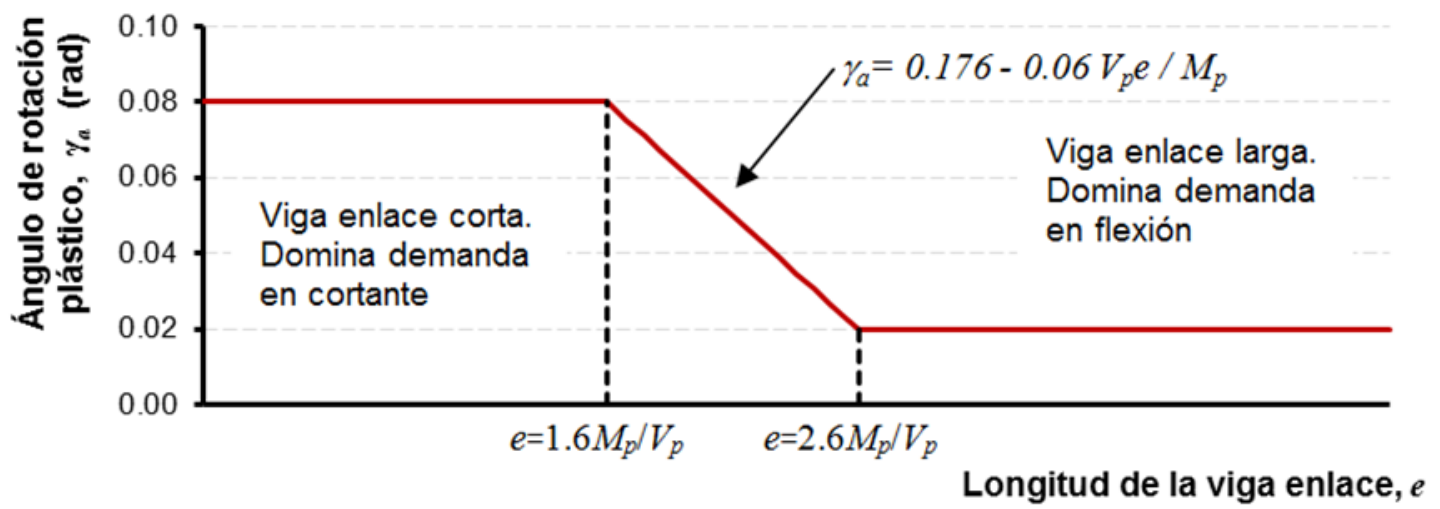

Figura 1. Ángulo de rotación plástico admisible en función de la longitud de la viga enlace

Algunas investigaciones recientes (Rozon et al. 2008; Koboevic y Olivia 2010) han evidenciado que edificios estructurados con marcos con contravientos excéntricos disipan energía de manera no uniforme en todos los niveles, donde la respuesta inelástica se concentra en los pisos inferiores, reportando daño por cortante o por flexión. Asimismo, los modelos de los edificios desarrollan ángulos de rotación inelásticos estables $\gamma_{p}$, con magnitudes que superan los límites de reglamentos especializados vigentes que se muestran en la figura 1.

Igualmente, los resultados de estudios experimentales (Kasai y Popov 1986; Popov y Engelhardt 1988; Stratan et al.2007; Dubina et al. 2008; Okazaki et al. 2009; Mansour 2010; Ji et al. 2014), que evaluaron el comportamiento de vigas enlace bajo cargas cíclicas para diferentes conexiones, longitudes y perfiles, coinciden en que las vigas enlace reportan ángulos de rotación inelásticos mayores a la normativa vigente. El modo de falla depende del detallado, protocolo de carga y las características de los especímenes; sin embargo, la respuesta de las vigas enlace en demandas relacionadas con el ángulo de rotación $\gamma_{p}$ en el límite normativo (figura 1) fue estable, sin fractura o fluencia del alma, fractura del patín o en algunos casos fractura en la conexión.

Este estudio pretende evaluar la capacidad y la respuesta inelástica de edificios estructurados con marcos con contraventeo excéntrico analizados y diseñados con la tendencia práctica y condiciones específicas del mercado mexicano. Los resultados se basan en análisis no lineales ante carga estática monótona creciente y un conjunto de análisis dinámicos a edificios de 6 y 9 pisos localizados en las condiciones de terreno blando de la Ciudad de México mediante modelos detallados. El estudio pretende aumentar el conocimiento adquirido, con el propósito de contribuir a establecer criterios simples que 
permitan al diseñador predecir con mayor certidumbre la capacidad inelástica y evolución del mecanismo de colapso de edificaciones con este sistema estructural.

\section{DESCRIPCIÓN DE LOS MODELOS}

Se estudiaron edificios de 6 y 9 niveles con alturas $H_{T}$ de 21.70 y 32.20 metros respectivamente, estructurados con marcos dúctiles de acero con contravientos excéntricos (figura 2). En el diseño se utilizó un factor de comportamiento sísmico $Q=4.0$, que es el máximo factor de ductilidad propuesto en las NTCDS-17 (2017). Los edificios regulares en planta, que se supusieron de uso de oficinas, se ubicaron en la Zona del Lago de la Ciudad de México con un periodo del suelo $T_{s}=2.0 \mathrm{~s}$. La configuración del modelo, el uso y el tipo de sección transversal se estableció mediante un muestreo de edificios existentes en el área metropolitana de la Ciudad de México, que puede consultarse en García (2015). Con base en la tendencia de la información recabada, en este estudio se decidió estudiar marcos con eslabones cortos cuyo comportamiento estuviera dominado por cortante.

El sistema de piso se consideró como un sistema compuesto formado con una lámina acanalada y un firme de concreto armado (conocida localmente como losacero), con una orientación que se varió por tablero para favorecer que la respuesta lateral en ambas direcciones fuera equivalente, por lo que igualmente se varió la dirección de las columnas de los marcos como se muestra en la figura $2 \mathrm{a}$.

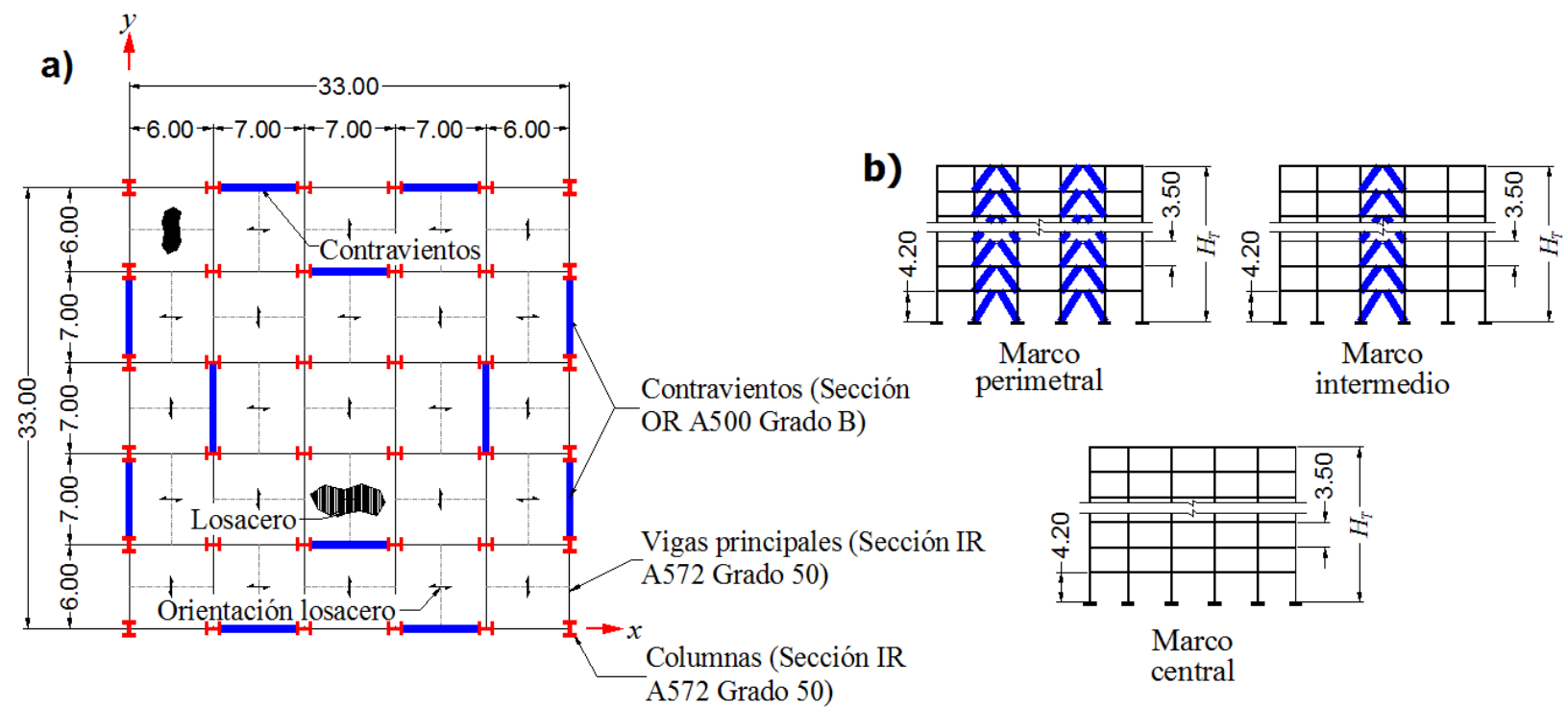

Figura 2. Modelo de 6 y 9 niveles: a) planta tipo, b) marcos que conforman el modelo

\section{Procedimiento de diseño}

En el diseño de los marcos momento-resistentes con contraventeo excéntrico, la viga enlace o eslabón de cortante es el elemento que constituye la primera línea de defensa; de manera que el resto de los elementos estructurales dependerá de su capacidad. En este proceso, el objetivo es producir un sistema que sea capaz de resistir las demandas sísmicas, mediante un mecanismo plástico consistente y estable.

En la filosofía de diseño por capacidad de marcos con contravientos excéntricos, el diseño queda gobernado por la configuración geométrica del marco y, por consiguiente, de la viga enlace (figura 3). Una vez que se predimensionan los elementos estructurales se deben aplicar las demandas gravitacionales (carga 
muerta más carga viva) más las demandas sísmicas aplicando las combinaciones de carga que correspondan. Con las demandas máximas, se realiza el diseño por resistencia de la viga enlace y, posteriormente, el resto de los elementos estructurales se deben diseñar con la máxima capacidad de los eslabones cuando se forma el mecanismo supuesto (figura 3). Finalmente, se recomienda verificar que la estructura cumpla con los estados límite de deformación.

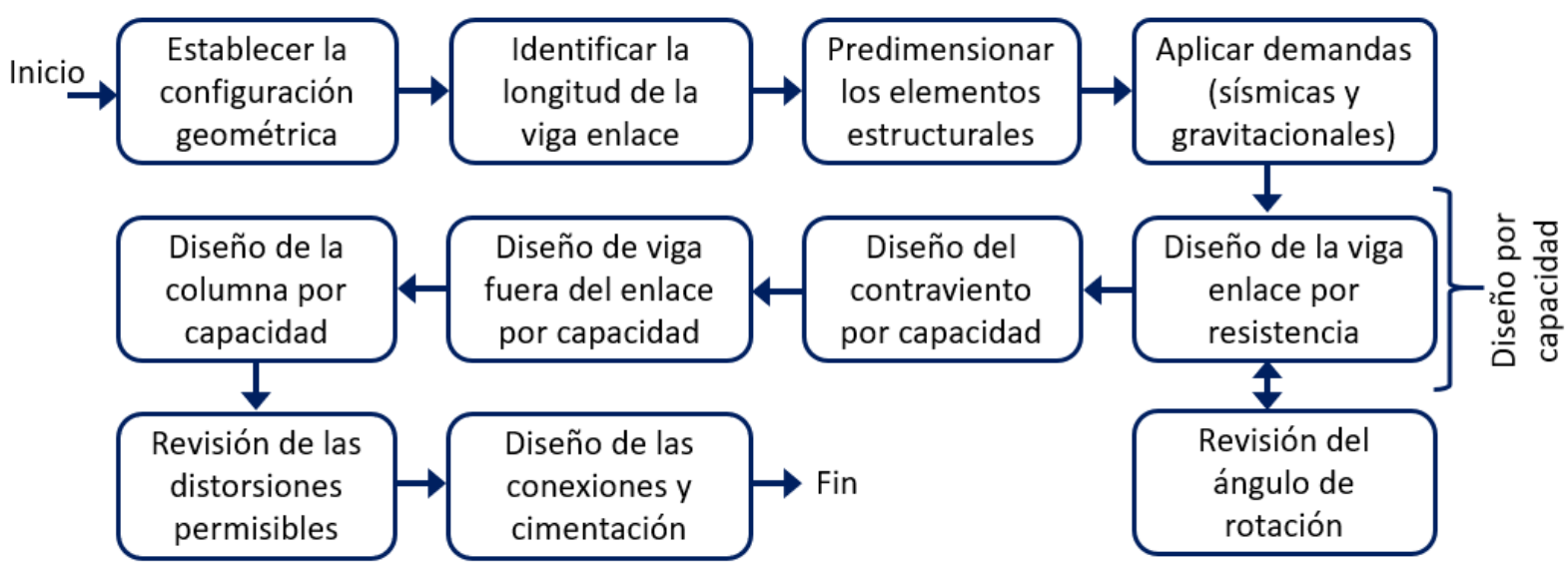

Figura 3. Filosofía de diseño por capacidad de marcos de acero dúctiles con contravientos excéntricos

En la tabla 1 se muestran las secciones finales del edificio de 6 niveles; mientras que en la tabla 2 se muestran las secciones del edificio de 9 niveles. En el diseño se consideró acero A572 Grado 50 con un $F_{y}=$ $3,515 \mathrm{~kg} / \mathrm{cm}^{2}$ en las vigas enlaces, vigas fuera del enlace y columnas; mientras que los contravientos se diseñaron con acero A500 Grado B con un $F_{y}=3,235 \mathrm{~kg} / \mathrm{cm}^{2}$. Con base en los análisis, el periodo fundamental del modelo de 6 pisos es $T_{6}=0.89 \mathrm{~s}$ y es igual a $T_{9}=1.06 \mathrm{~s}$ en el modelo de 9 pisos.

En la práctica local, $I R d \times \mathrm{x} w$ se refiere a una viga de sección I laminada con un peralte nominal $d$ en milímetros y un peso lineal $w$ en $\mathrm{kg} / \mathrm{m}$. Además, $O R d$ x $d \times$ x $e$ se refiere a un elemento de sección cuadrada hueca con un peralte $d$ y un espesor de la placa $e$, ambos en milímetros.

Tabla 1. Secciones transversales del edificio de 6 niveles $(\mathrm{mm}, \mathrm{kg} / \mathrm{m})$

\begin{tabular}{|c|c|c|c|c|c|c|}
\hline \multirow[b]{2}{*}{ Piso } & \multicolumn{4}{|c|}{ Marcos con contravientos excéntricos } & \multicolumn{2}{|c|}{ Marcos momento-resistente } \\
\hline & Viga enlace & $\begin{array}{l}\text { Viga fuera del } \\
\text { enlace }\end{array}$ & Contravientos & Columnas & Viga & \\
\hline N6 & IR 356x56.7 & IR 356x56.7 & OR $203 \times 203 \times 9.5$ & $610 \times 217.8$ & IR 457x68.4 & IR 610x195 \\
\hline N5 & IR $356 \times 101.3$ & IR $356 \times 101.3$ & OR $305 \times 305 \times 12.7$ & IR 610 & IR $533 x$ & 195 \\
\hline N4 & IR 406x & IR $406 x$ & OR & IR 610 & IR 533 & 195 \\
\hline N3 & IR $406 \times 148.9$ & IR 406x148.9 & OR $406 \times 406 \times 15.9$ & IR 686x500 & IR $533 \times 74.4$ & IR $610 \times 195$ \\
\hline N2 & IR 406x 148.9 & IR 406x148.9 & OR 406x406x15.9 & IR 686x500 & IR $610 \times 82.0$ & IR $610 \times 195$ \\
\hline N1 & IR $457 \times 144.3$ & IR $457 \times 144.3$ & OR $406 \times 406 \times 15.9$ & IR $686 \times 500$ & IR $610 \times 82.0$ & IR 610x195 \\
\hline
\end{tabular}

En las previsiones sísmicas del AISC 341-10 y las NTC-DCEA-17, el ángulo de rotación plástico deberá ser menor o igual a 0.08 rad para las vigas enlace cortas; mientras que para vigas enlace largas, la magnitud del ángulo de rotación no deberá de exceder de 0.02 rad. Para vigas enlaces intermedias, deberá 
de interpolarse la magnitud del ángulo de rotación. En García y Tapia (2014) es posible encontrar una discusión sobre el propósito de estos límites.

Tabla 2. Secciones transversales del edificio de 9 niveles $(\mathrm{mm}, \mathrm{kg} / \mathrm{m})$

\begin{tabular}{|c|c|c|c|c|c|c|}
\hline \multirow[b]{2}{*}{ Piso } & \multicolumn{4}{|c|}{ Marcos contraventeados excéntricamente } & \multicolumn{2}{|c|}{ Marcos momento-resistente } \\
\hline & Viga enlace & $\begin{array}{c}\text { Viga fuera del } \\
\text { enlace }\end{array}$ & Contravientos & Columnas & Vigas & \\
\hline N9 & & IR 356x56.6 & & & IR $457 \times 81.9$ & IR 533x165.3 \\
\hline N8 & & & & & & \\
\hline N7 & & & & & & \\
\hline N6 & & & & & & \\
\hline N5 & & & & & & 95.0 \\
\hline N4 & $356 \times 101.3$ & & OR 3 & & IR 533 & 195.0 \\
\hline N3 & IR 406x114.7 & & & & IR 610 & IR 6 \\
\hline $\mathrm{N} 2$ & & & & & & \\
\hline N1 & IR 406x114.7 & IR 406x 114.7 & OR $355 \times 355 \times 15.9$ & IR 762x531.4 & IR $610 \times 81.9$ & IR $610 \times 308.2$ \\
\hline
\end{tabular}

En la figura 4 se muestra la revisión de desplazamientos en el estado límite de servicio, el estado límite de seguridad contra colapso y la revisión de los ángulos de rotación máximos por entrepiso en las direcciones globales. El diseño estructural de marcos contraventeados excéntricamente depende altamente de la respuesta de la viga enlace, que es gobernada por la restricción del ángulo de rotación plástico $\gamma_{p}=0.08$ rad (AISC 341-10, NTC-DCEA-17). Con base en los resultados, este límite (figura 4c) rige el diseño aún sobre las revisiones de distorsión de entrepiso.

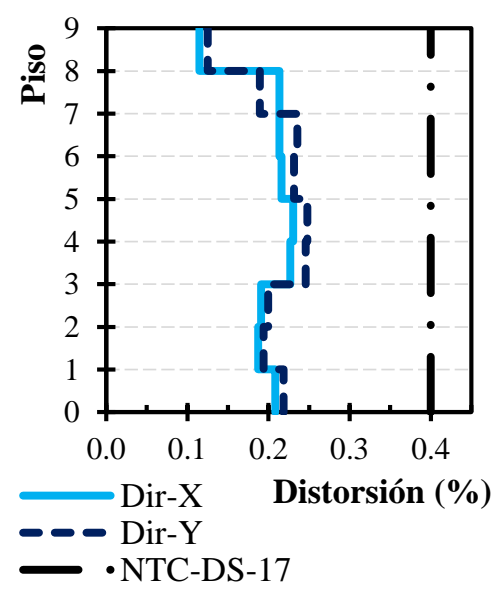

a) Estado límite de servicio

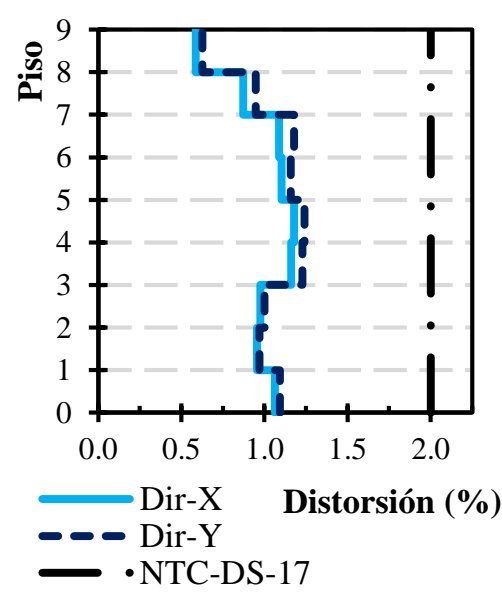

b) Prevención de colapso

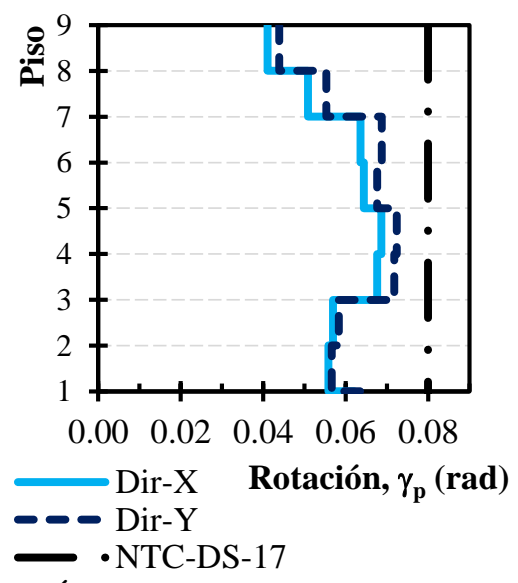

c) Ángulos de rotación máximos

Figura 4. Revisión de los estados límites del modelo de 9 pisos

\section{MODELADO INELÁSTICO}

Se realizaron análisis inelásticos tridimensionales en el programa OpenSees (Mazzoni et al., 2006) siguiendo el criterio de modelado que se muestra en la figura 5. Las propiedades del material se modelaron utilizando el modelo propuesto por Giuffre-Menegotto-Pinto (GMP) con el fin de obtener una representación realista que incluyera el endurecimiento por deformación y el efecto Bauschinger. 


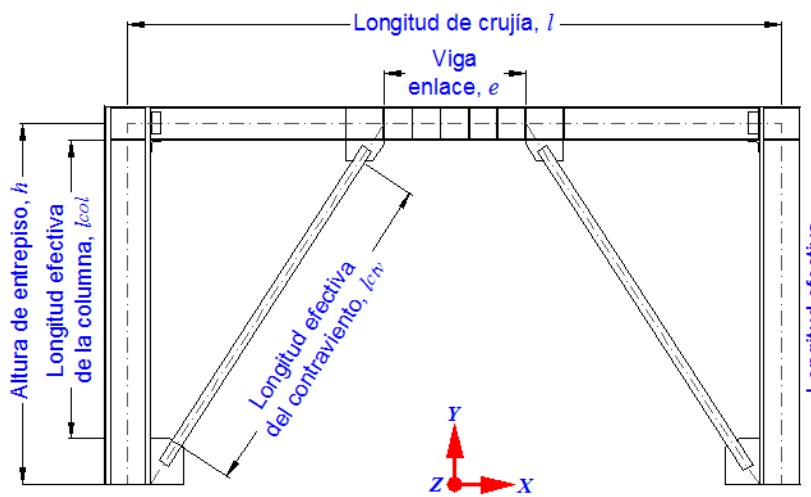

a) Elevación del marco

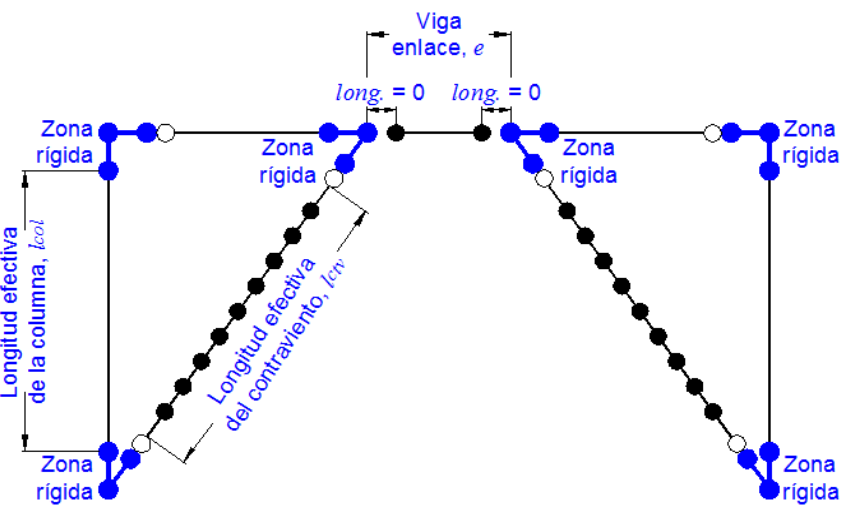

b) Modelo estructural

Figura 5. Vista en elevación y condiciones de modelado del marco contraventeado excéntricamente

Los perfiles estructurales se modelaron con elementos que pueden desarrollar plasticidad a lo largo de su longitud, donde las secciones transversales se discretizaron mediante segmentos rectangulares fraccionados en fibras (figura 6a). La cantidad de fibras se definió mediante un estudio paramétrico para optimizar los resultados y el tiempo de cómputo. Es posible consultar mayor información en Terzic (2013), García (2015) y Tapia et al. (2016). En los análisis, los contravientos se modelaron mediante diez subelementos para incluir los efectos de segundo orden, donde el pandeo se modeló hacia fuera del plano. Los contravientos están biarticulados y fueron unidos a zonas rígidas que tienen diez veces las propiedades de los elementos que convergen a las uniones. Además, los contravientos están concebidos con una imperfección inicial al centro del elemento con una magnitud $L / 1000$ (figura $6 \mathrm{~b}$ ) con una configuración parabólica. Esta magnitud es la máxima permitida en manuales especializados (IMCA, 2014) y pretende incluir errores en la fabricación de los perfiles, el montaje en obra y/o daño durante el transporte.

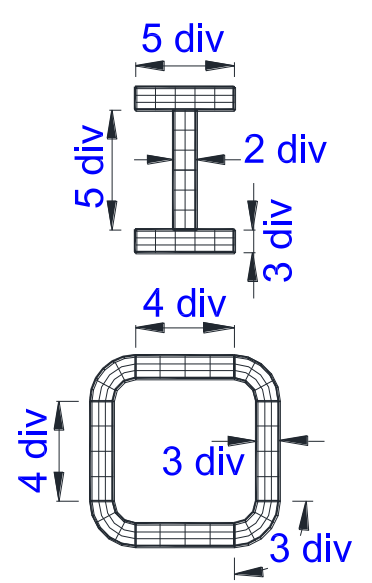

a) Secciones transversales

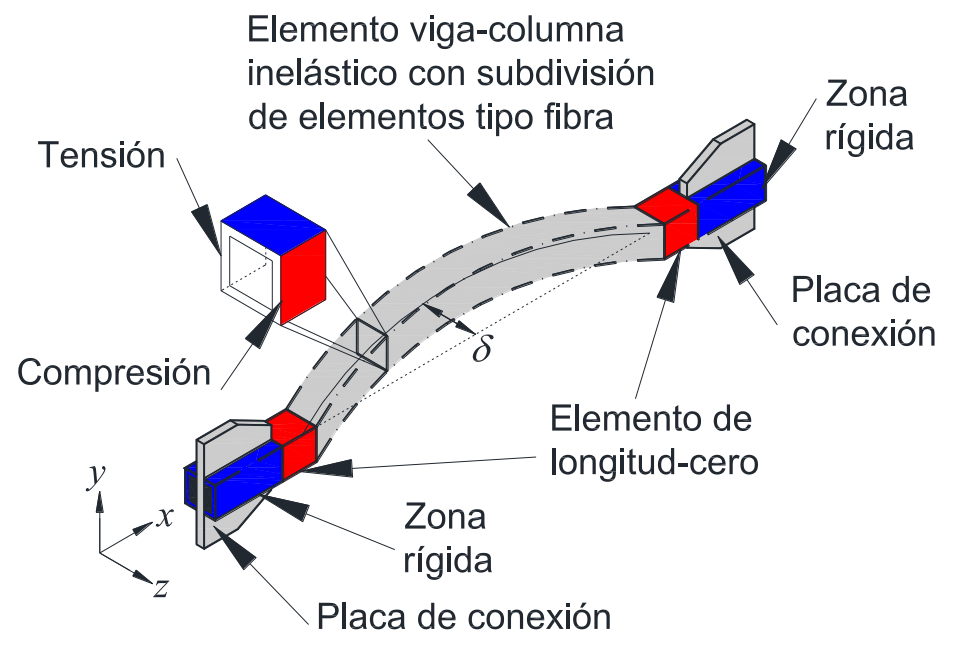

b) Imperfección inicial del contraviento

Figura 6. Características de los modelos en estudio

Adicionalmente, los edificios considerados se analizaron con un desplome lateral igual a $H / 500$, donde $H$ es la altura del entrepiso, que es el límite de los manuales especializados (IMCA, 2014). Este desplome (figura 7) pretende simular las imperfecciones en las conexiones, elementos estructurales, errores 
en la cimentación, entre otros, con el propósito de obtener resultados analíticos que tengan congruencia con las condiciones reales de los edificios.

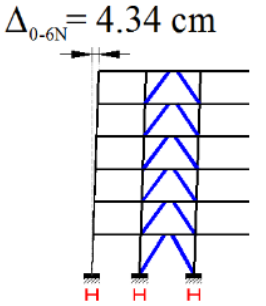

a) Modelo de 6 niveles

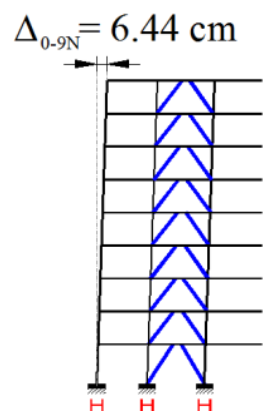

b) Modelo de 9 niveles

Figura 7. Imperfección en los modelos por efecto del desplome vertical

\section{Modelo inelástico de la viga enlace}

La viga enlace de cortante es el punto crítico en el diseño y detallado de los marcos con contraventeo excéntrico; donde el resto de los elementos estructurales y las conexiones se diseñan para permanecer prácticamente elásticos. Con el objetivo de que el comportamiento del eslabón concuerde con el comportamiento de investigaciones analíticas y experimentales que se tomaron como referencia (e.g. Richards y Uang 2004; Rozon et al. 2008; Mansour 2010, entre otras), se incluyó un modelo detallado en la conexión de la viga enlace (figura 7). Consulte una discusión más amplia sobre la filosofía de diseño por capacidad en Berman y Bruneau (2006); Bosco y Rossi (2009); García y Tapia (2014); SMIE (2017).

En algunas investigaciones (por ejemplo, Prinz 2010), se ha modelado la viga enlace como un elemento con resortes traslacionales y rotacionales en los extremos. Sin embargo, en este estudio debido a que se consideró una viga enlace corta, en la conexión del enlace a la viga fuera del enlace se consideró un elemento de longitud cero unido únicamente con resortes traslacionales en paralelo (figura 8a); de modo que se transmiten a la viga fuera del enlace únicamente demandas por cortante. La rigidez de los resortes se estableció como se explica en la figura 8b, con base en estudios analíticos previos (Ramadan y Ghobarah 1995; Prinz 2010).

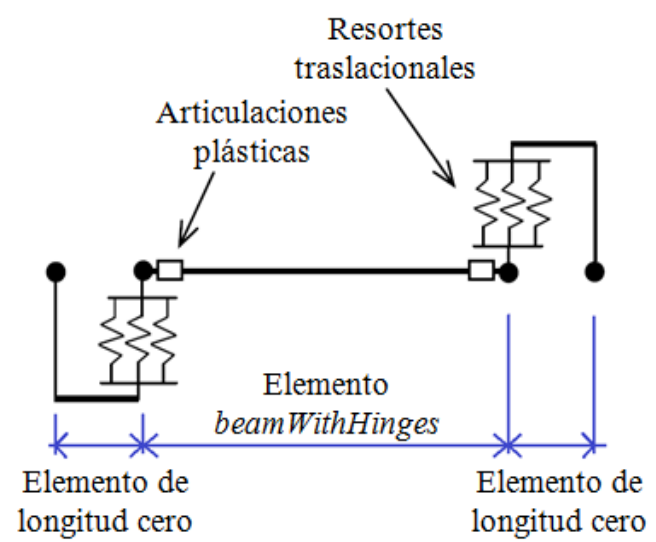

a) Subelementos del modelo de conexión de la viga enlace

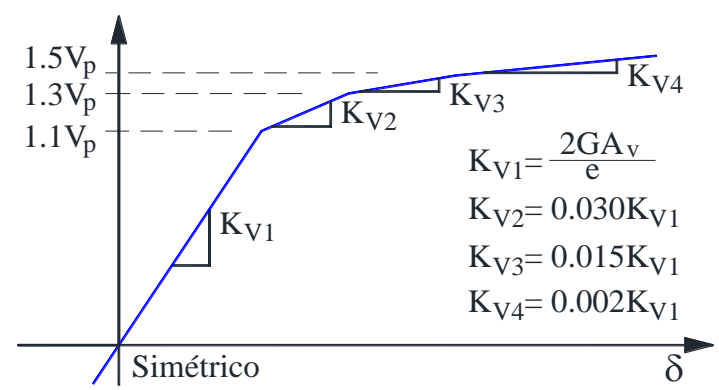

b) Definición de la rigidez de los resortes traslacionales (Prinz 2010)

Figura 8. Modelo de la viga enlace 


\section{Esfuerzos de fluencia reales del acero estructural}

En las NTC-DCEA-17, la resistencia esperada de un elemento estructural se define mediante el factor $R_{y}$ que establece la sobrerresistencia del material en función del tipo de acero y está definida como la proporción entre el esfuerzo de fluencia real y el esfuerzo de fluencia teórico. En esta investigación se consideraron los resultados de investigaciones recientes (García 2015; Tapia et al. 2016) que centraron la atención en determinar la sobrerresistencia del material en perfiles y placas disponibles en el mercado local. Esta decisión obedece a que la magnitud del factor $R_{y}$ estipulada en las NTC-DCEA-17 está basada íntegramente en el AISC 341-10, que no es (ni tendría por qué ser) una buena representación de las condiciones y características de los perfiles y placas disponibles en el mercado mexicano.

Con base en los resultados, la media muestral del factor de sobrerresistencia del material relacionada con el esfuerzo de fluencia del acero A572 Grado 50 (NMX B-284 Grado 50) fue igual a $R_{y}=1.20\left(F_{y \mu}=\right.$ $4,223 \mathrm{~kg} / \mathrm{cm}^{2}$ ), que se obtuvo mediante 809 certificados de perfiles IR. Además, el factor para el acero A500 Grado B (NMX B-199) fue igual a $R_{y}=1.22\left(F_{y \mu}=3,955 \mathrm{~kg} / \mathrm{cm}^{2}\right)$, considerando los resultados de 92 certificados disponibles. En la figura 9 se muestra la proporción de los esfuerzos obtenidos en los certificados de acero A572 Grado 50, el histograma asociado usando una distribución de Gumbel y los límites normativos (NTC-DCEA-17) para ejemplificar el procedimiento. Asimismo, se muestra el histograma que se calculó en el estudio estadístico, donde se supuso una distribución log-normal. Es posible conocer más información sobre la sobrerresistencia del material en placas y perfiles de acero en México en García (2015) y Tapia et al. (2016).

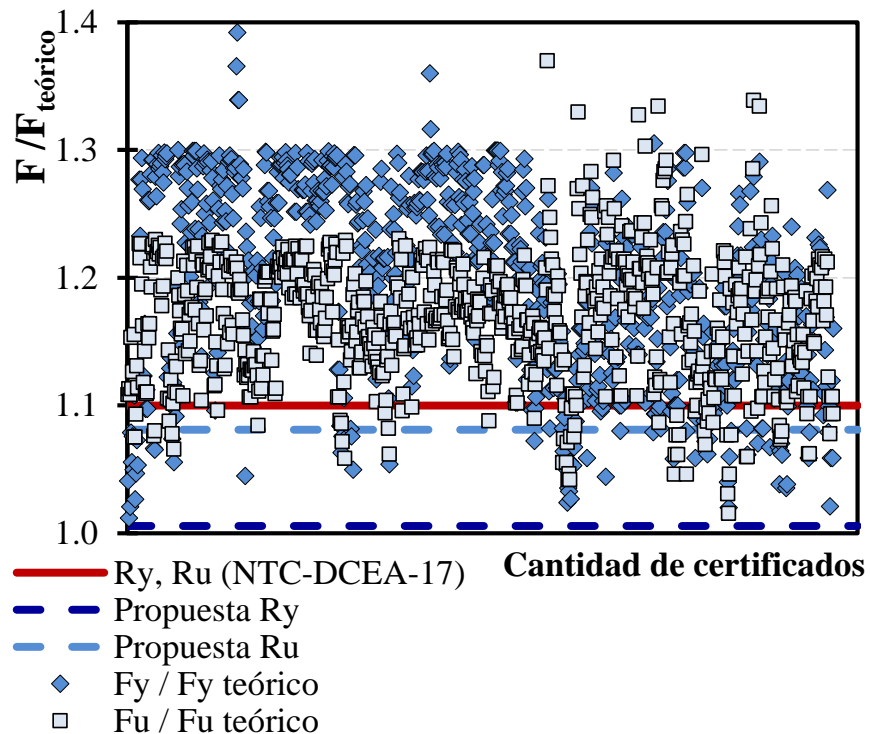

a) Proporción entre el esfuerzo de los certificados y el esfuerzo teórico

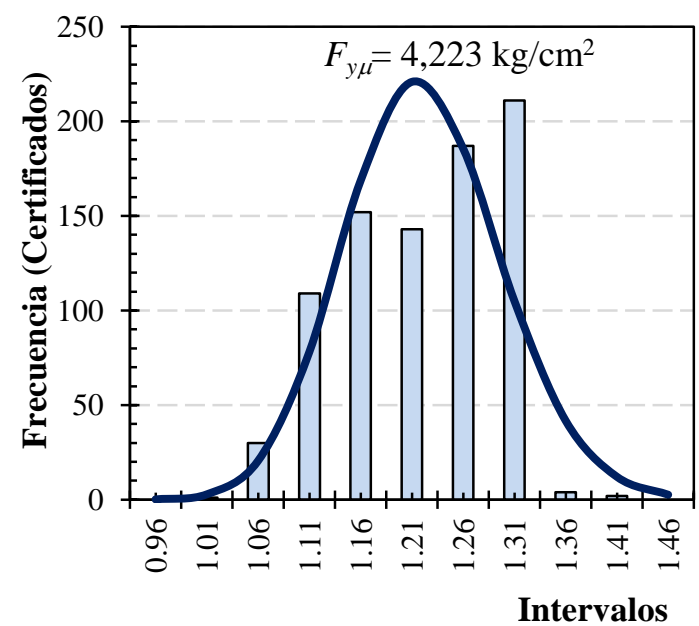

b) Histograma calculado

Figura 9. Sobrerresistencia del acero ASTM-A572 Grado 50 (NMX B-284 Grado 50) en el mercado local

\section{ANÁLISIS ESTÁTICOS NO LINEALES}

Conforme al FEMA-356 (2000), para un análisis estático inelástico es común usar un patrón de cargas triangular invertido, que corresponde aproximadamente a la respuesta del primer modo fundamental de la 
estructura. De hecho, Gutiérrez y Tapia, 2014 demostraron que edificios regulares estructurados con marcos de acero de baja y mediana altura son mayormente excitados por patrones de carga relacionados con el modo fundamental.

En este artículo se discuten los resultados de la dirección global $x$, pero se hace notar que la respuesta en ambas direcciones principales fue equivalente debido a su regularidad. Con el propósito de evaluar la contribución total de las fuerzas laterales entre los marcos en una dirección, los marcos del modelo se conectan entre sí mediante elementos infinitamente rígidos y se incluyeron los efectos de un diafragma rígido en cada nivel (figura 10). Adicionalmente, en los análisis no lineales se consideró un amortiguamiento viscoso igual a $\zeta=0.05$ conforme a la definición de los espectros de diseño sísmico (e.g. MDOC-08 2008).

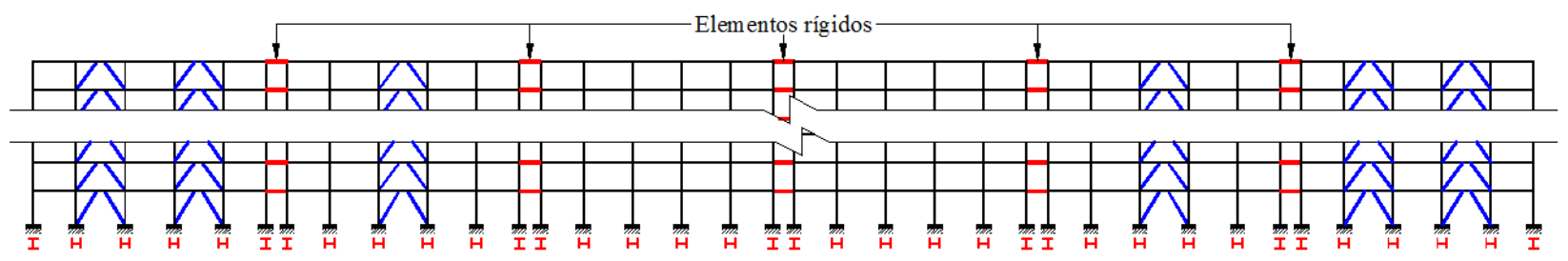

Figura 10. Configuración de los modelos discretizados en OpenSees

En el post-proceso de los resultados de los análisis no lineales ante carga estática monótona creciente, el último paso se definió mediante un estudio estadístico (García 2015) sobre la magnitud del ángulo de rotación inelástico reportado en pruebas experimentales de vigas enlace (Dubina et al. 2008, Okazaki et al. 2009; Mansour 2010; Ji et al. 2014). En el proceso, se consideraron pruebas experimentales que evaluaron el comportamiento de vigas con características equivalentes a las propuestas en este estudio. En ellas, los ángulos de rotación inelásticos de las vigas enlace se definieron como el modo de falla que induce la demanda máxima que puede soportar el elemento por fractura en el alma, fractura en el patín, etc. Entonces, en los análisis de esta investigación, la máxima capacidad inelástica no está definida como una solución matemática, con base en el paso que numéricamente el programa puede calcular. Esta decisión pretende evitar que los resultados estén basados en el posible colapso matemático, sino que se definió considerando la capacidad que pueden desarrollar las secciones transversales que conforman el modelo.

Como se muestra en la figura 11, la media muestral del estudio, que puede ser consultado en García (2015) resultó ser igual a $\mu=0.154$ rad. Conservadoramente, aquí se consideró una media muestral menos una desviación estándar que resultó ser igual a $\mu-\sigma=0.127 \mathrm{rad}$ (figura 11), que es mayor al límite normativo $\gamma=0.08$ rad. Considerar está magnitud del ángulo de rotación máximo en las vigas enlace permitirá relacionar los resultados analíticos con las capacidades de edificios reales con mayor certidumbre. Es posible consultar más información sobre el procedimiento empleado, una amplia discusión de estos resultados de pruebas experimentales y sobre el estudio estadístico en García (2015). 


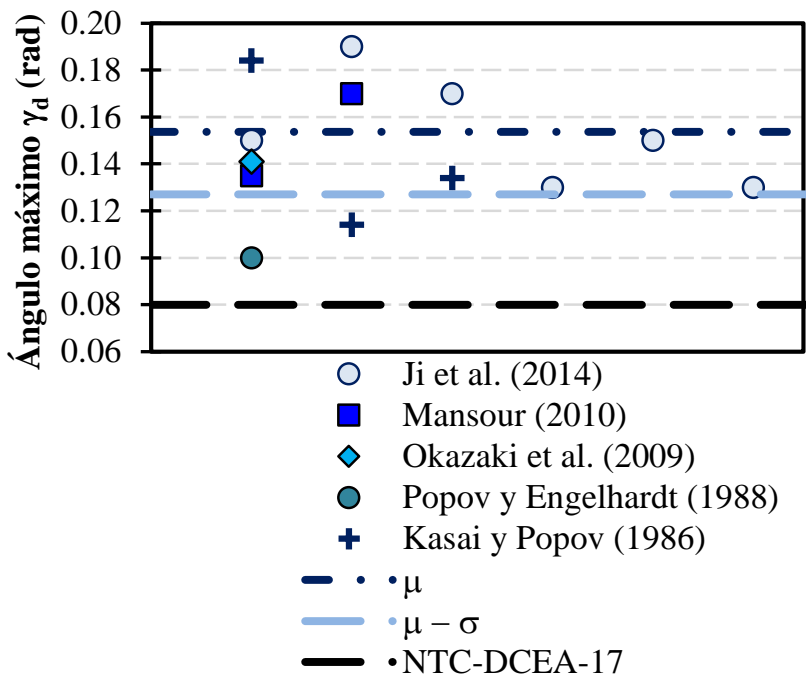

a) Capacidades reportadas en pruebas experimentales

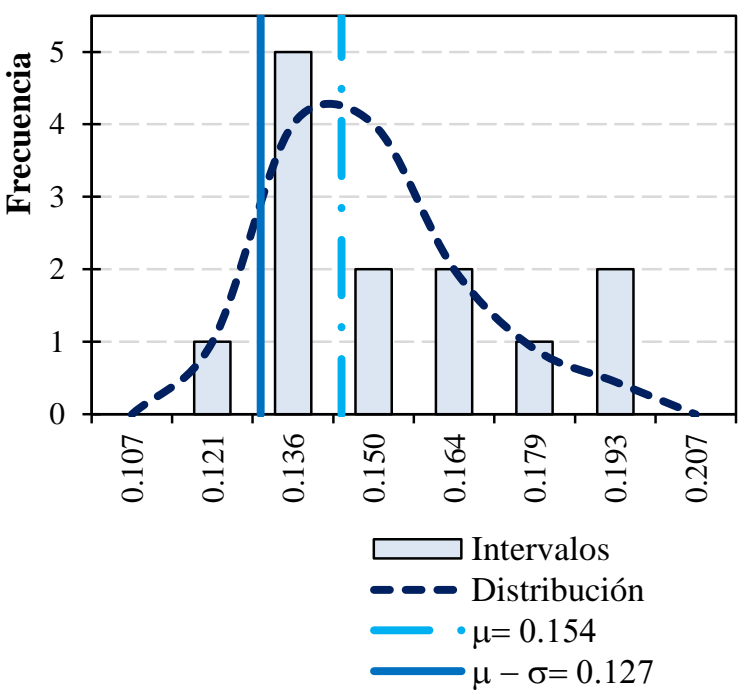

b) Histograma

Figura 11. Ángulos de rotación en vigas enlaces reportadas en pruebas experimentales

\section{Ductilidad y sobrerresistencia}

El comportamiento de los modelos se analizó por medio de curvas normalizadas cortante - distorsión de entrepiso y curvas globales. En la figura 12 se muestran las curvas globales de los modelos estudiados, donde el cortante $V$ está normalizado con el peso total de la estructura $W_{T}$ y la distorsión (definida como el desplazamiento en la azotea dividida entre la altura del edificio) está en porcentaje.

Las curvas de capacidad son típicas de modelos discretizados en fibras, donde la plastificación paulatina de las fibras ocasiona cambios de pendiente muy ligeros entre pasos contiguos reportados en los análisis estáticos no lineales, puesto que reportan la fluencia sucesiva de fibras en la sección transversal. Este efecto ocasiona que sea complicado establecer el punto de fluencia de la estructura, porque el inicio de la plasticidad de algunas fibras no puede ser asociado con la fluencia de la sección transversal. Por consiguiente, en este estudio se estableció la fluencia como el paso donde la pendiente tangencial entre los puntos fuera mayor al 5 por ciento de la rigidez elástica como se muestra en la figura 12.

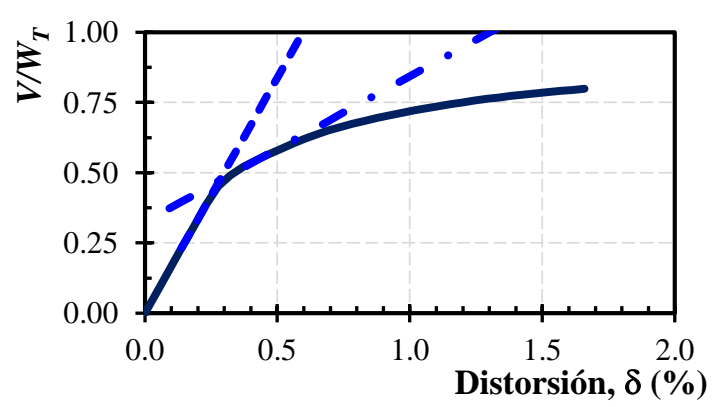

a) Modelo de 6 niveles

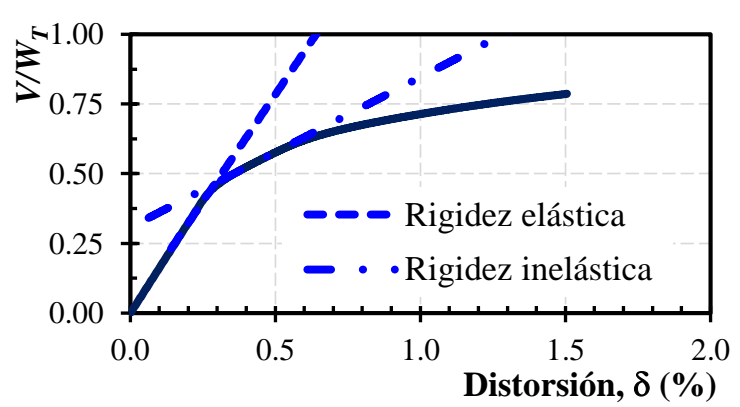

b) Modelo de 9 niveles

Figura 12. Curvas globales cortante - distorsión de los modelos de estudio 
Los resultados obtenidos están relacionados con una concentración de daño en los pisos inferiores como se ejemplifica en las curvas de capacidad obtenidas para el modelo de seis pisos (figura 13). Esto concuerda con otras investigaciones (Tapia y Gutiérrez 2016; Rozon et al. 2008; Koboevic y Olivia 2010), donde la disipación de energía no es uniforme en todos los niveles de los modelos, debido a que únicamente las vigas enlace de los entrepisos inferiores o intermedios fluyen por cortante y redistribuyen la demanda a los elementos adyacentes concentrando el daño.
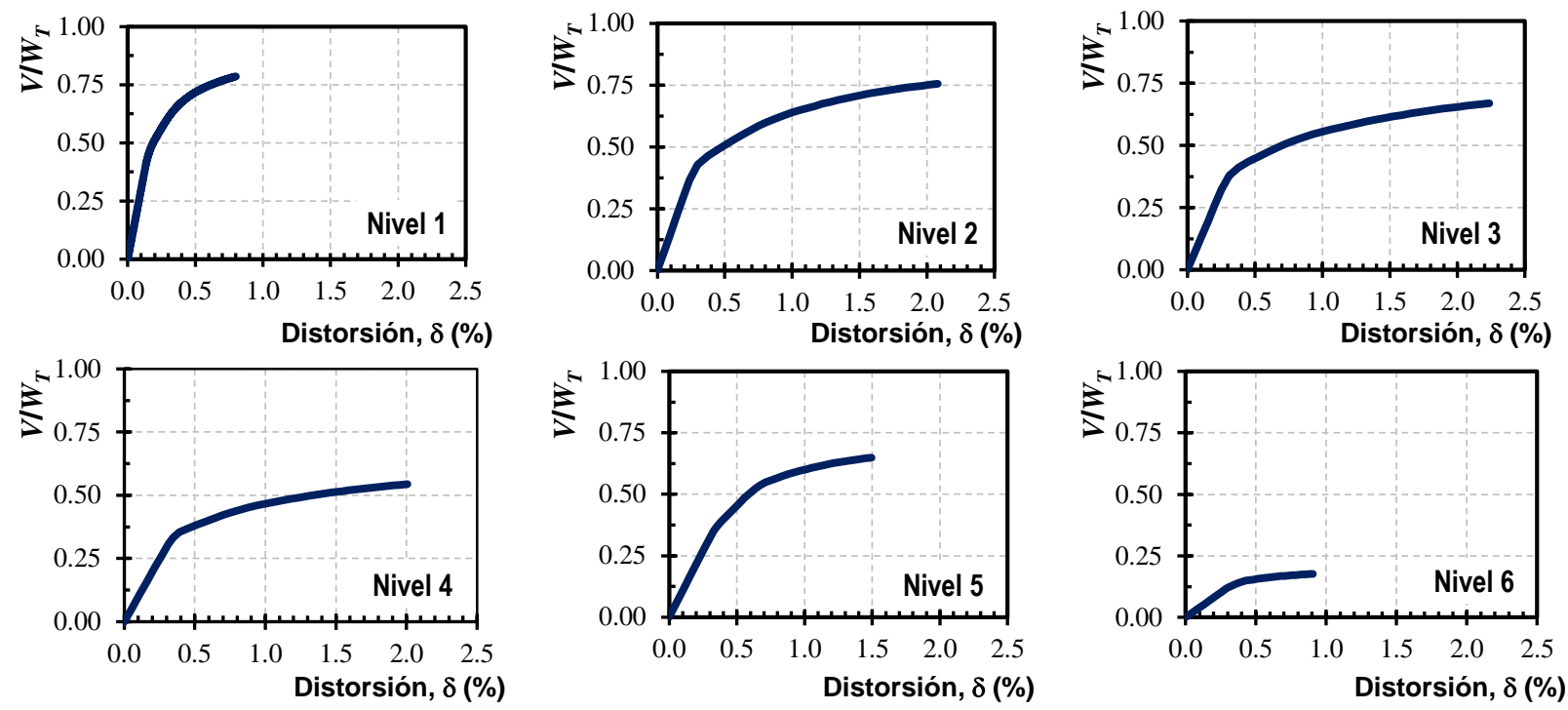

Figura 13. Curvas de entrepiso cortante - distorsión del edificio de 6 niveles

Con las curvas de capacidad, se determinaron las capacidades inelásticas de los modelos estudiados (tabla 3), La ductilidad desarrollada se definió como la proporción entre la distorsión última $\delta_{u}$ y la distorsión de fluencia $\delta_{y}$; mientras que la capacidad de sobrerresistencia se definió como la proporción entre el cortante máximo $V_{u}$ dividido entre el cortante de diseño de la etapa elástica $V_{d}$.

Tabla 3. Capacidades de los modelos de estudio

\begin{tabular}{lcc}
\hline Modelo & Edificio 6 niveles & Edificio 9 niveles \\
\hline Distorsión de fluencia, $\delta_{y}(\%)$ & 0.277 & 0.288 \\
Distorsión última, $\delta_{u}(\%)$ & 1.659 & 1.506 \\
Cortante de fluencia, $V_{y} / W_{T}$ & 0.452 & 0.450 \\
Cortante último, $V_{u} / W_{T}$ & 0.799 & 0.786 \\
Ductilidad, $\mu=\delta_{u} / \delta_{y}$ & 5.992 & 5.224 \\
Sobrerresistencia, $\Omega=V_{u} / V_{d}$ & 1.766 & 1.748 \\
\hline
\end{tabular}

La ductilidad que desarrollaron los edificios es superior a la ductilidad objetivo en la etapa de diseño elástico $(Q=4)$. Los resultados denotan una tendencia, donde los modelos de menor altura son capaces de desarrollar una respuesta más dúctil, que había sido identificada previamente por Tapia y Tena (2013) y que no está explícitamente incluida en la reglamentación especializada. 
Por otra parte, la capacidad de sobrerresistencia de los edificios resultó ser menor a la que propone la reglamentación vigente. Los resultados no tienen dependencia de la altura de los modelos. Algunas investigaciones como Hamid (2011), señalan que la sobrerresistencia global de los marcos contraventeados excéntricamente tiene una alta dependencia de la respuesta de la viga enlace, por lo que sería necesario realizar un mayor número de análisis antes de hacer alguna recomendación normativa.

\section{Capacidad de deformación}

En la figura 14 se muestran las distorsiones por entrepiso, distorsiones globales reportadas en los análisis y el límite propuesto en las NTC-DS-17 para la revisión del estado límite de servicio. En ambos casos, la distorsión máxima de entrepiso es cercana al límite permisible propuesto $\left(\delta_{y p}=0.4 \%\right)$, por esta razón, el límite normativo parece adecuado, pese a que las distorsiones de los entrepisos intermedios del modelo de 9 niveles exceden el límite en un 5\%

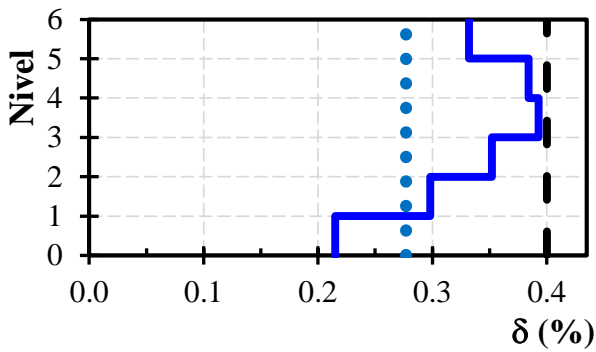

a) Modelo de 6 pisos

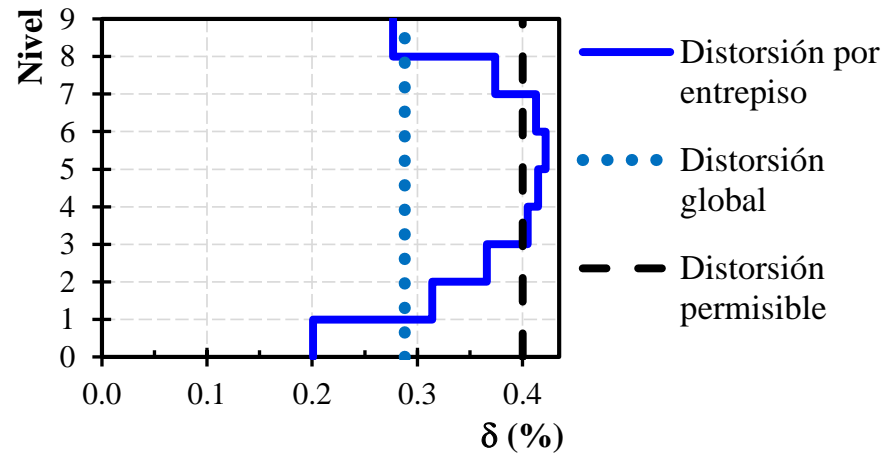

b) Modelo de 9 pisos

Figura 14. Perfil de distorsiones en el estado límite de servicio

Por otra parte, en la figura 15 se muestran los resultados de la revisión en el estado límite de seguridad contra colapso. Ambos modelos denotan una concentración de daño en los pisos intermedios, donde la magnitud máxima reportada en los resultados del modelo de 9 niveles difiere en un $15 \%$ al límite propuesto en las NTC-DS-17 igual a 2.0\%. Así, los límites normativos parecen una buena estimación de la capacidad de deformación de este sistema estructural.

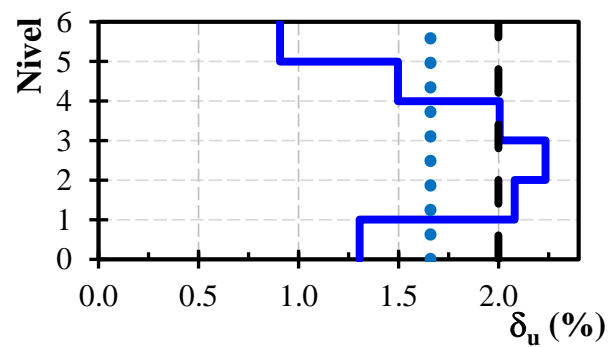

a) Edificio de 6 pisos

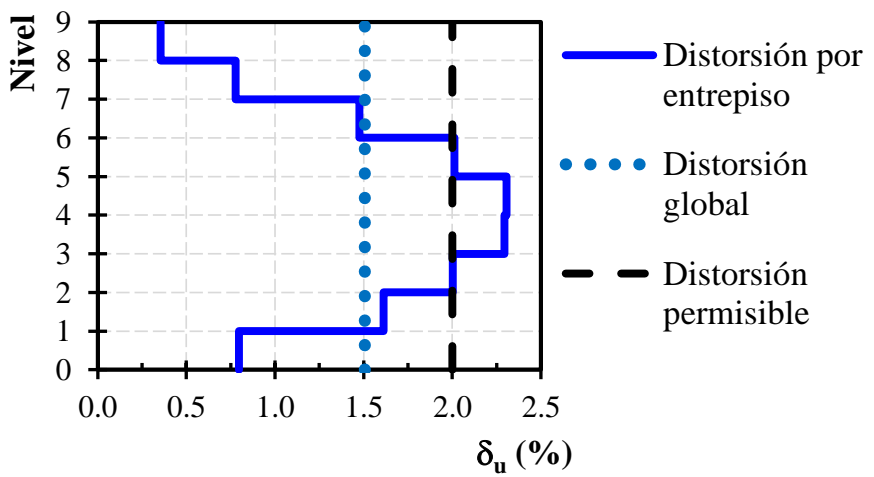

b) Edificio de 9 pisos

Figura 15. Perfil de distorsiones en el estado límite de prevención de colapso 


\section{Ángulos de rotación plástico, $\gamma_{p}$}

En la figura 16 se muestran los ángulos de rotación inelástico máximos desarrollados por entrepiso en los análisis de los modelos de 6 y 9 niveles. Con base en los resultados, el ángulo de rotación plástico supera la magnitud propuesta en los reglamentos vigentes (CNBC-05; AISC 341-10; NTC-DS-17) igual a $\gamma_{\mathrm{p}}=0.08$ rad. Adicionalmente, en la figura 16 se hace notar el límite propuesto en esta investigación basado en investigaciones experimentales igual a $\gamma_{\mu-\sigma}=0.127 \mathrm{rad}$.

Con base en los resultados, los límites normativos para estimar el ángulo de rotación parecen ser conservadores. De hecho, esta observación coincide con los resultados de otras investigaciones analíticas como Rozon et al. (2008) y Koboevic y Olivia (2010). Los criterios vigentes en la normativa internacional sobre la respuesta de marcos con contravientos excéntricos están basados en estudios experimentales realizados por Popov (Roeder y Popov 1978; Malley y Popov 1984; Kasai y Popov 1986; Popov y Engelhardt 1988).

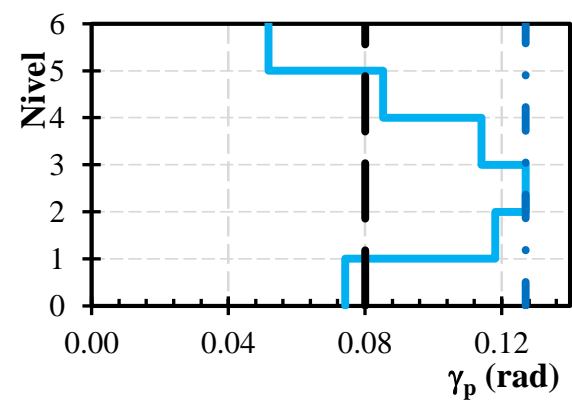

a) Edificio de 6 niveles

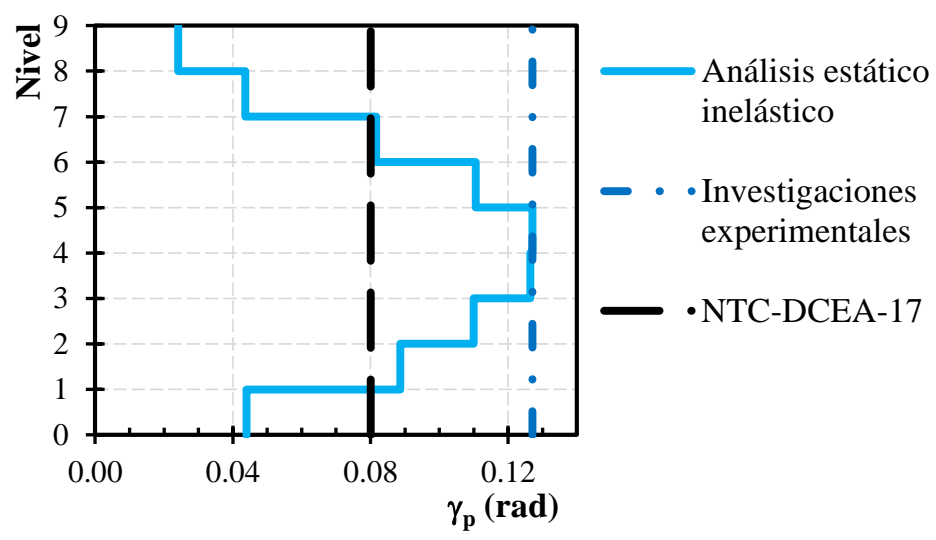

b) Edificio de 9 niveles

Figura 16. Perfiles de los ángulos de rotación plástico

\section{ANÁLISIS DINÁMICOS NO LINEALES}

Se realizaron análisis dinámicos no lineales, donde se seleccionaron diez acelerogramas históricos (tabla 4) registrados en la Ciudad de México conforme a la Base Mexicana de Sismos Fuertes (2000). En particular, se seleccionaron registros que tuvieran demandas de aceleración máxima en el intervalo de periodos cercano al periodo fundamental de los modelos de este estudio como se muestra en la figura 17.

Particularmente en este estudio, los registros se escalaron con el propósito de que la aceleración espectral máxima fuera equivalente a la demanda del espectro de diseño para que correspondieran al mismo peligro sísmico. Con este propósito se consideraron los criterios de las NTC-DS-17 para la selección de los registros, donde se solicita que las formas en el tiempo de las amplitudes y de los contenidos de frecuencia sean congruentes con las observadas para la fuente y sitio que conducen a la intensidad considerada. Esos acelerogramas se afectan por factores de escalamiento que conducen a que las ordenadas de los espectros que miden la intensidad de las excitaciones sísmicas bajo consideración correspondan al espectro de diseño para periodos comprendidos entre 0.2 y 1.3 veces el periodo natural del modo dominante de vibrar de la estructura que está incluido en la figura 17. 
Tabla 4. Estaciones y acelerogramas seleccionado

\begin{tabular}{clclccc}
\hline No Nombre & $\begin{array}{c}\text { Fecha de } \\
\text { ocurrencia }\end{array}$ & Tipo de suelo & Identificación & $\begin{array}{c}\mathrm{S}_{\mathrm{a} \mathrm{máx}} \\
\left(\mathrm{cm} / \mathrm{s}^{2} / \mathrm{g}\right)\end{array}$ & $\begin{array}{c}\text { Factor } \\
\text { de escala }\end{array}$ \\
\hline 1 & Alberca olímpica & $14 / 09 / 95$ & Estratificado & AO249509 & 0.234 & 7.34 \\
2 & Jardines de Coyoacán & $15 / 06 / 99$ & Arcilloso, Zona lago & JC549906 & 0.198 & 4.44 \\
3 & Cibeles & $25 / 04 / 89$ & Blando, arcillas & CI058904 & 0.303 & 6.48 \\
4 & Mariano Escobedo & $14 / 09 / 95$ & Estratificado & ME529509 & 0.209 & 7.48 \\
5 & Granjas & $25 / 04 / 89$ & Arcilla, limo, arena & GR278904 & 0.379 & 7.89 \\
6 & Ángel Urraza & $25 / 04 / 89$ & Estratificado & AU468904 & 0.264 & 8.53 \\
7 & Córdoba & $25 / 04 / 89$ & Alto riesgo sísmico & CO568904 & 0.229 & 5.75 \\
8 & Plutarco Elías Calles & $25 / 04 / 89$ & Arcilloso, Zona lago & PE108904 & 0.275 & 6.20 \\
9 & Colegio Madrid & $31 / 05 / 90$ & Blando, arcillas & DFCM9005 & 0.319 & 27.30 \\
10 & Lindavista & $25 / 04 / 89$ & Blando, arcillas & LV178904 & 0.237 & 7.24 \\
\hline
\end{tabular}
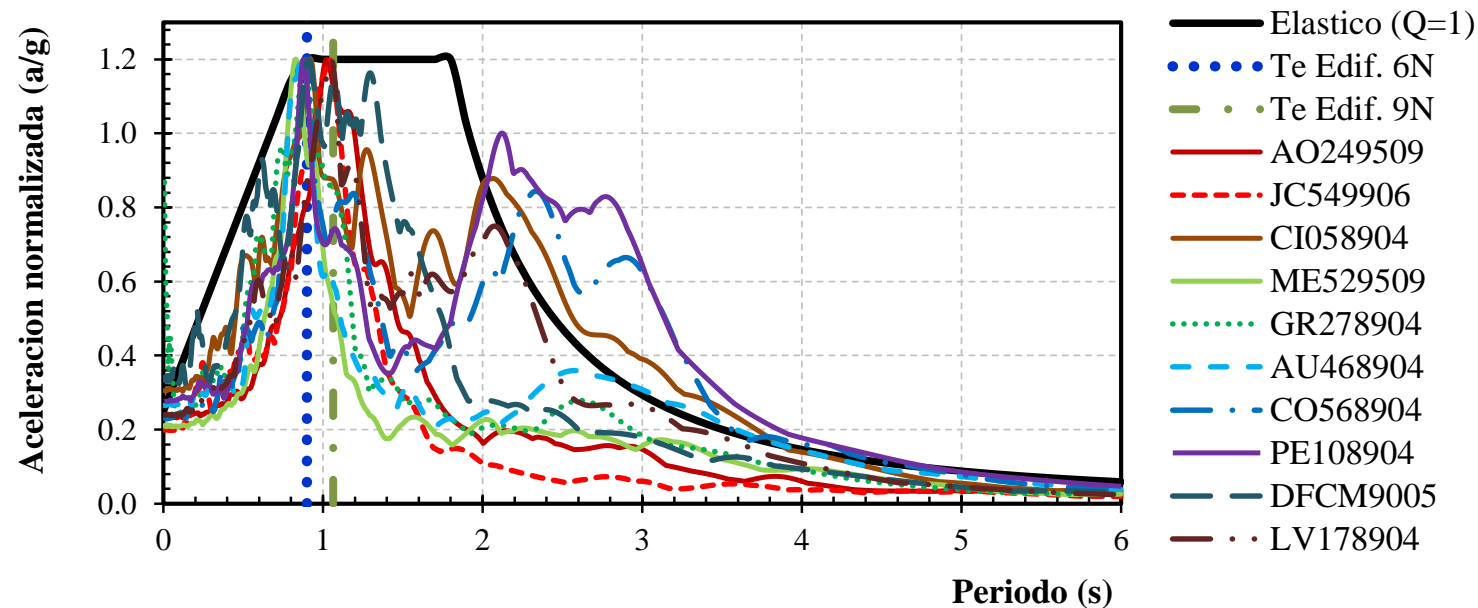

Figura 17. Comparativa entre los espectros de respuesta y el espectro de diseño elástico

En la figura 18 se muestran algunos de los diagramas de histéresis (cortante normalizado con el peso total contra distorsión global) del modelo de seis pisos con las demandas de aceleración escaladas con el propósito de mostrar el procedimiento empleado en esta investigación.
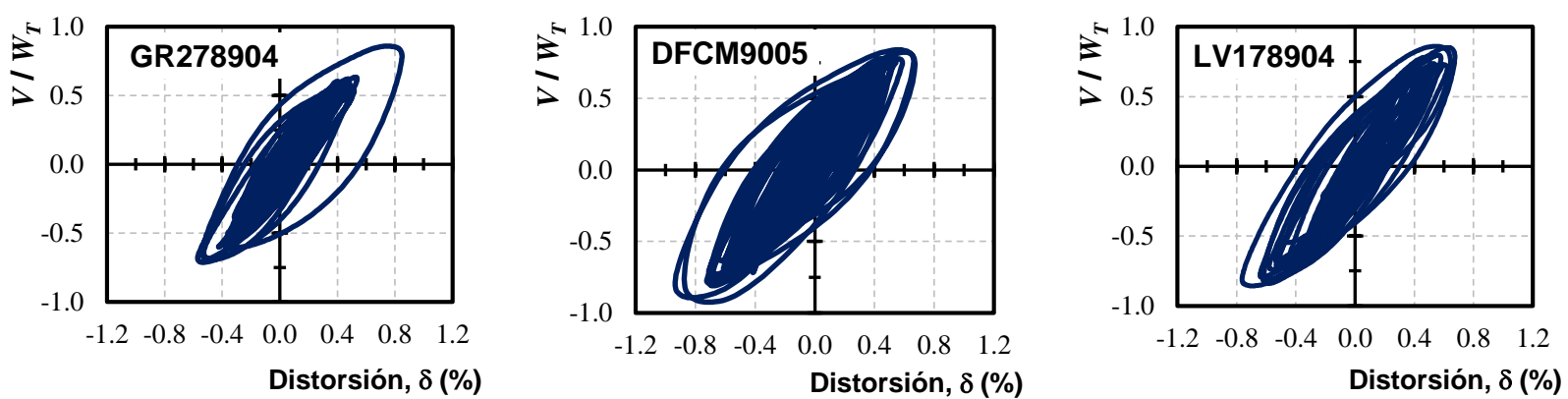

Figura 18. Curvas de histéresis globales representativas de la respuesta global del modelo de 6 niveles 
En la tabla 5 se muestran las distorsiones de fluencia obtenidas en los análisis donde la magnitud media es igual a $0.45 \%$ y $0.38 \%$ que es muy cercana al límite normativo igual a $0.40 \%$ (NTC-DS-17). Como se esperaba, ninguno de los modelos colapsó, por lo que en la tabla se muestra la distorsión máxima desarrollada por los modelos. Dado que las demandas están muy alejadas de la restricción de desplazamiento al estado límite de colapso, los resultados indican una reserva de capacidad para las demandas impuestas.

Tabla 5. Distorsiones de fluencia y máxima por acelerograma

\begin{tabular}{lcccc}
\hline \multirow{2}{*}{ Registro } & \multicolumn{2}{c}{ Edificio 6 niveles } & \multicolumn{2}{c}{ Edificio 9 niveles } \\
\cline { 2 - 5 } & $\delta_{\mathrm{y}}(\%)$ & $\delta_{\text {máx }}(\%)$ & $\delta_{\mathrm{y}}(\%)$ & $\delta_{\operatorname{máx}}(\%)$ \\
\hline AO249509 & 0.38 & 0.79 & 0.39 & 0.68 \\
JC549906 & 0.46 & 0.62 & 0.34 & 0.53 \\
CI058904 & 0.45 & 1.19 & 0.40 & 0.88 \\
ME529509 & 0.0 & 0.63 & 0.34 & 0.48 \\
GR278904 & 0.48 & 0.85 & 0.35 & 0.67 \\
AU468904 & 0.44 & 0.81 & 0.34 & 0.56 \\
CO568904 & 0.44 & 0.80 & 0.41 & 0.65 \\
PE108904 & 0.45 & 0.83 & 0.38 & 0.66 \\
DFCM9005 & 0.47 & 0.94 & 0.48 & 0.85 \\
LV178904 & 0.51 & 0.76 & 0.46 & 0.69 \\
\hline Promedio & 0.45 & 0.82 & 0.39 & 0.66
\end{tabular}

\section{Ángulo de rotación inelástico, $\gamma_{p}$}

En la figura 19 se muestra el comportamiento de las vigas enlace por entrepiso del modelo de 9 pisos ante el acelerograma LV178904. En la figura se incluye la rotación de diseño propuesta en los reglamentos especializados $\gamma_{p}=0.08$ rad y el ángulo de rotación que está basado en los resultados de pruebas experimentales igual a $\gamma_{\mu-\sigma}=0.127 \mathrm{rad}$, que se discutió en secciones previas. Las curvas de histéresis coinciden con los resultados de pruebas experimentales a vigas enlace dúctiles (e.g. Okazaki et al. 2009).

Con base en los resultados, las vigas enlace de prácticamente todos los entrepisos de ambos modelos reportan una respuesta inelástica. Sin embargo, se nota que los pisos intermedios tienen una concentración del daño que supera ampliamente la rotación normativa $\left(\gamma_{p}=0.08 \mathrm{rad}\right)$ y cuya magnitud máxima es cercana al ángulo de rotación considerado en este estudio $\left(\gamma_{\mu-\sigma}=0.127 \mathrm{rad}\right)$. Estos resultados concuerdan con otras investigaciones analíticas como Richards y Uang (2004), Rozon et al. (2008) y Koboevic y Olivia (2010) y con estudios experimentales como Mansour (2010) y Ji et al. (2014), donde las vigas enlace de modelos de edificios con marcos con contravientos excéntricos desarrollan rotaciones plásticas en ciclos estables con magnitudes que superan el límite normativo (AISC 314-10; NTC-DCEA-17). 

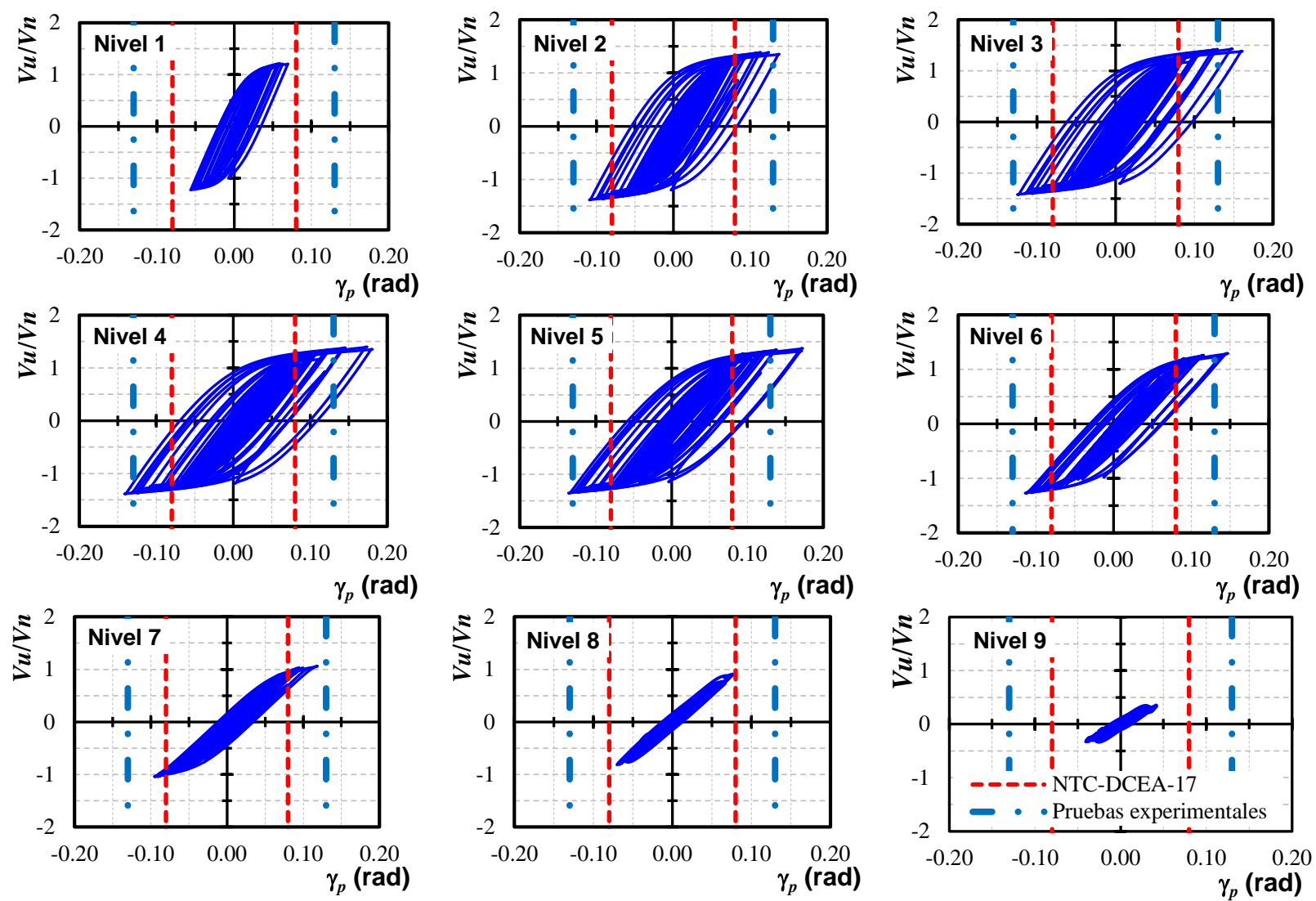

Figura 19. Comportamiento histerético de vigas enlace del edificio de 9 pisos ante el registro LV178904

En la figura 20 se muestran los perfiles de los ángulos de rotación de los análisis dinámicos inelásticos (ADI) en forma estadística. La línea ADI (Promedio) establece el promedio de los ángulos de rotación de las vigas enlace considerando los resultados de los diez acelerogramas; la línea ADI (Máximo) establece la magnitud máxima que se desarrolló ante alguno de los acelerogramas y como magnitud constante se muestra el límite de las NTC-DCEA-17 (0.08 rad) y la magnitud de la rotación de las pruebas experimentales discutida anteriormente $(0.127 \mathrm{rad})$.

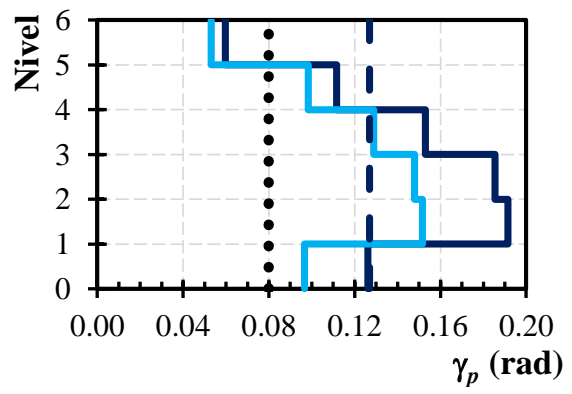

a) Edificio de 6 niveles

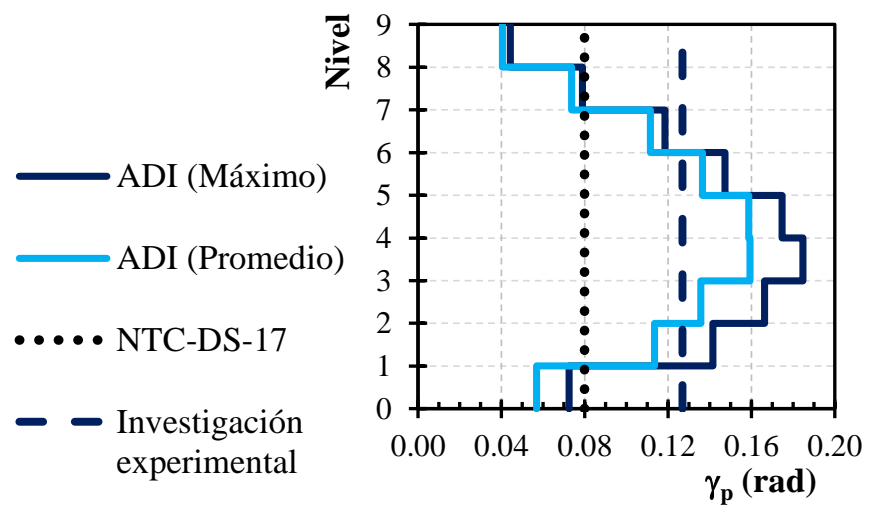

b) Edificio de 9 niveles

Figura 20. Ángulos de rotación reportados en los análisis dinámicos inelásticos 
Con base en los resultados, los modelos disipan energía a través de la fluencia de las vigas enlace. Sin embargo, se nota una acumulación del daño en los pisos inferiores e intermedios, pese a que el diseño siguió estrictamente la filosofía de un diseño por capacidad basado en los criterios de la reglamentación vigente. El límite propuesto para estimar la rotación de las vigas enlace parece ser una referencia adecuada de la capacidad que pueden desarrollar los eslabones por cortante con perfiles IR de marcos con contraventeo excéntrico dúctil en forma estable.

\section{CONCLUSIONES}

En esta investigación se evalúa la respuesta de modelos de edificios de seis y nueve pisos estructurados con marcos contraventeados excéntricamente. La configuración, el uso y el tipo de sección transversal de los elementos estructurales se establecieron a través de un muestreo de los edificios más usados en el área metropolitana de la Ciudad de México. Los modelos de edificios de 6 y 9 pisos se diseñaron estrictamente siguiendo la filosofía de diseño por capacidad.

Se realizaron análisis ante carga estática monótona creciente y análisis dinámicos inelásticos paso a paso en el programa de análisis OpenSees (Mazzoni et al. 2006). En los análisis se incluyeron los resultados de un muestreo de la sobrerresistencia del material que reportan los perfiles de acero disponibles en el mercado mexicano, a partir de los esfuerzos de fluencia reportados en certificados de calidad de laboratorio. Además, la capacidad inelástica máxima considerada en el post-proceso de resultados no se asoció con el último paso que matemáticamente puede desarrollar el programa, sino que la distorsión se limitó al ángulo de rotación plástica que reportan pruebas experimentales disponibles en la literatura. En los análisis dinámicos inelásticos se emplearon diez acelerogramas históricos registrados en la Ciudad de México, que se seleccionaron por corresponder al peligro sísmico supuesto en la etapa de diseño. Las principales contribuciones de esta investigación son las siguientes:

- En la etapa del análisis elástico, el diseño queda regido por las restricciones del ángulo de rotación sugerido en los reglamentos especializados para la viga enlace; de modo, que los modelos satisfacen fácilmente las restricciones de desplazamiento relativo de los entrepisos de las NTC-DS-17.

- Las distorsiones obtenidas en los análisis estáticos no lineales para la revisión del estado límite de servicio y de prevención contra colapso son aceptablemente parecidas a las magnitudes propuestas en las NTCS-DS-17.

- La ductilidad desarrollada por los modelos $\left(\mu_{6}=5.99\right.$ y $\left.\mu_{9}=5.22\right)$ es superior a la que se estimó en la etapa de diseño $(Q=4)$, lo que implica que los edificios con este sistema estructural pueden desarrollar una ductilidad mayor a la estimada. Los resultados denotan una posible dependencia de la altura de los edificios.

- La sobrerresistencia que desarrollan los edificios estudiados $\left(\Omega_{6}=1.77\right.$ y $\left.\Omega_{9}=1.75\right)$ es menor a la magnitud supuesta, pese a que los modelos tienen una sobrerresistencia del material igual a $R_{y}=1.20$ para acero A572 Grado 50 determinada de un muestreo de certificados de calidad comentada anteriormente.

- En los análisis dinámicos no lineales, las vigas enlace desarrollan ángulos de rotación plástico superiores al sugerido en la reglamentación especializada. Esto implica que las vigas enlace de los edificios estudiados fueron capaces de disipar más energía que la estimada, a través del daño, en forma estable.

- Pese a que la gran mayoría de las vigas enlace fluyen, los resultados indican una concentración de daño en los pisos intermedios e inferiores que no depende de la altura de los modelos. Esta tendencia se reporta en los modelos estudiados, pese a que en el diseño se aplicó estrictamente un diseño por capacidad riguroso y amerita un estudio detallado de la redistribución de carga como parte de futuras investigaciones. 


\section{AGRADECIMIENTOS}

Los autores agradecen el apoyo otorgado al primer autor al Consejo Nacional de Ciencia y Tecnología (CONACYT) por la beca otorgada para los estudios de maestría.

\section{REFERENCIAS}

AISC 341-16 (2016), "Seismic provisions for structural steel buildings", American Institute of Steel Construction, Chicago, IL.

Base Mexicana de Sismos Fuertes. (2000). Comite de la Base Nacional de datos de sismos fuertes, CDROM, Volumen 2.

Berman J. y Bruneau M. (2006), "Experimental and analytical investigation of tubular braced frames", Engineering Structures, No. 29. Pp. 1929 - 1938. https://doi.org/10.1016/j.engstruct.2006.10.012

Bosco M. y Rossi P.P. (2009), "Seismic behavior of eccentrically braced frames", Engineering Structures, No. 31, pp. 664-674. https://doi.org/10.1016/j.engstruct.2008.11.002

CNBC-05. (2005), National Building Code - Canada 2005. Volume 1. Canadian Committee of Building Codes and Fire Prevention. National Council Research, 12th. Ed. Ottawa, ON

Dubina D., Stratan A. y Dinu F. (2008). Dual high-strength steel eccentrically braced frames with removable links, Earthquake Engineering and Structural Dynamics, Vol. 37, pp. 1703-1720. https://doi.org/10.1002/eqe.828

FEMA-356 (2000). Prestandard and Commentary for the Seismic Rehabilitation of Building, Federal Emergency Management Agency, Washintong, D.C.

García J. S. (2015). Respuesta inelástica de edificios regulares estructurados con marcos dúctiles de acero, Tesis de Maestría, Posgrado en Ingeniería Estructural, Universidad Autónoma Metropolitana, Unidad Azcapotzalco, Septiembre. México.

García J. S. y Tapia E. (2014). Observaciones sobre el diseño de la viga enlace en marcos de acero con contraventeo excentrico", Memorias XIX Congreso Nacional de Ingenería Sísmica, Sociedad Mexicana de Ingeniería Estructural. Puerto Vallarta, Jal.

Gutiérrez I. y Tapia E. (2014). Influencia de los modos superiores en la respuesta inelástica de edificios estructurados con marcos dúctiles de acero, Memorias XIX Congreso Nacional de Ingenería Sísmica, Sociedad Mexicana de Ingeniería Estructural. Puerto Vallarta, Jal.

Hamid S. (2011). Ductility and ultimate strength of eccentric braced frame. International Conference on Advanced Materials Engineering, IPCSIT, Vol. 15, pp. 68-74.

IMCA. (2014), Manual de Construcción en Acero, Instituto Mexicano para la Construcción en Acero (IMCA), 5a. Edición, Ed. Limusa. México. 
Ji X., Ma Q., Wang Y. y Okazaki T. (2014). Ciclyc behavior of steel shear links used in replaceable coupling beams, 10h U.S. National Conference on Earthquake Engineering, EERI, Memorias, Anchore, Alaska, July.

Kasai K. y Popov E. (1986). Cyclic web buckling control for shear link beams, Journal of Structural Engineering, Vol. 112, pp. 505-523. https://doi.org/10.1061/(ASCE)0733-9445(1986)112:3(505)

Koboevic S. y Olivia S. (2010). Design and seismic behavior of taller eccentrically braced frames, Canadian Journal of Civil Engineering. Vol. 37, pp. 195-208. https://doi.org/10.1139/L09-131

Malley J.O. y Popov E.P. (1984), "Shear links in eccentrically braced frames", Journal of Structural Engineering. ASCE, Vol. 110. No. 9, pp. 2275-2295. https://doi.org/10.1061/(ASCE)07339445(1984)110:9(2275)

Mansour N. (2010). Development of the design of eccentrically braced frames with replaceable shear links, Tesis Doctoral, Departamento de Ingeniería Civil, Universidad de Toronto.

Mazzoni S., McKenna F., Scott M. y Fenves G. (2006). Open system for earthquake engineering simulation, user command-language manual, Report NEES grid-TR 2004-21. Pacific Earthquake Engineering Research, University of California. Berkeley, CA. http://opensees.berkeley.edu.

MDOC-08 (2008). Comisión Federal de Electricidad - Instituto de Investigaciones Eléctricas, Capitulo de Diseño por Sismo, Manual de Diseño de Obras Civiles. México.

NTC-DCEA-17 (2017). Normas Técnicas Complementarias para el Diseño y Construcción de Estructuras de Acero. En Gaceta Oficial de la Ciudad de México. No. 220Bis. Vigésima época. Gobierno de la Ciudad de México. Diciembre.

NTC-DS-17 (2017). Normas Técnicas Complementarias sobre Diseño por Sismo. En Gaceta Oficial de la Ciudad de México. No. 220Bis. Vigésima época. Gobierno de la Ciudad de México. Diciembre.

Okazaki T., Engelhardt M. D., Drolias A., Schell E., Hong J. K. y Uang C. M. (2009). Experimental investigation of link-to-column connections in eccentrically braced frames, Journal of Construction Steel Research, Vol. 65, pp. 1401 - 1412. https://doi.org/10.1016/j.jcsr.2009.02.003

Popov E. P. y Engelhardt M. D. (1988). Seismic eccentrically braced frames, Journal of Constructional Steel Research, Vol. 10, pp. 321-354. https://doi.org/10.1016/0143-974X(88)90034-X

Prinz G. (2010), "Using buckling-restrained braces in eccentric configurations", Tesis Doctoral, Departamento de Ingeniería Civil y Ambiental, Universidad Brigham Young

Ramadan T. y Ghobarah A. (1995), “Analytical model for shear-link behavior”, Journal of Structural Engineering, ASCE. Vol. 121, No. 11, pp. 1574-1580. DOI: 10.1061/(ASCE)07339445(1995)121:11(1574)

Richards, P. y Uang, C. M. (2004). Development of testing protocol for links in eccentrically braced frames, 13th World Conference on Earthquake Engineering, Vancouver, B.C., Canada.

Roeder C.W. y Popov E.P. (1978), "Eccentrically braced Steel frames for earthquakes", Journal of Structural Engineering. ASCE, Vol. 110. No. 2, pp. 340-354. https://doi.org/10.1016/01410296(83)90034-2 
Rozon J., Kovoebic S. y Tremblay R. (2008). Study of global behavior of eccentrically braced frames in response to seismic loads, 14th World Conference on Earthquake Engineering, Beijing, China. Octubre.

Stratan A., Dogariu A. y Dubina D. (2007), Bolted links for eccentrically braced frames: influence of link stiffness, Proc. of the Fifth AISC / ECCS International Workshop "Connections in Steel Structures V. Behaviour, Strength \& Design", June 3-5, 2004. Ed. F.S.K. Bijlaard, A.M. Gresnigt, G.J. van der Vegte. Delft University of Technology, The Netherlands. ISBN: 978-90-9019809-5, pp. 223-232.

Terzic V. (2013). Modeling SCB frame using beam - columns elements. Seminario de OpenSees, presentación. http://opensees.berkeley.edu/

Tapia E. y Tena A. (2013). Diseño sísmico de marcos de acero contraventeados. Parte 2: Evaluación de la metodología. Revista de Ingeniería Sísmica. Vol. 88, pp. 69-90.

Tapia E., García S. y Del Rincón A. (2016). Estudio paramétrico del modelado de contravientos de acero. Revista de Ingeniería Sísmica 94, pp. 49-74.

Tapia E. y Gutiérrez S. (2016), "Respuesta inelástica de marcos dúctiles de acero ante patrones de carga". Revista Internacional de Ingeniería de Estructuras. Vol. 21. No. 1. Pp. 105-122. 